\title{
Global Academic Journal of Medical Sciences
}

Available online at www.gajrc.com

DOI: 10.36348 /gajms.2021.v03i04.001

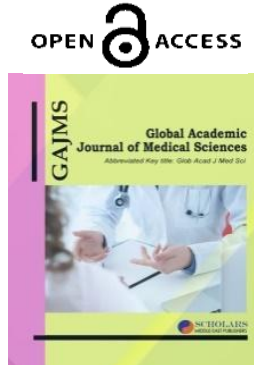

ISSN: $2706-9028(0)$

ISSN: 2707-2533 (P)

Case Report

\section{Gastric and Rectal Metastases of Breast Invasive Lobular Carcinoma Occurring 12 Years after Initial Treatment: Case Report and Literature Review}

\author{
Gustave Negamiyimana ${ }^{*}$, Justin Bagorane ${ }^{1}$, Nadin Shawar AL Tamimi $^{1}$, Mohamed El Fadli $^{1}$, Rhizlane \\ Belbaraka1 \\ ${ }^{1}$ Department of Medical Oncology, Mohammed VI Hospital University; CADI Ayyad University, Marrakech, Morocco
}

\section{*Corresponding Author \\ Gustave Negamiyimana}

Article History

Received: 08.06.2021

Accepted: 05.07.2021

Published: 12.07.2021

\begin{abstract}
Metastases of breast cancer to the gastrointestinal tract (GIT) are rare. We here report of a 52-year-old woman, in whom breast invasive lobular carcinoma (ILC) metastasized to the stomach and rectum more than 10 years later. Gastric and rectal metastasis became evident after 12 and 13 years, respectively, of the initial treatment of breast cancer. Immunohistochemistry and histological examination confirmed the mammary origin of the digestive malignancy, because of the histopathological similarities between these metastatic lesions and the initial breast cancer. We chose chemotherapy for these metastatic lesions. Treatment should be decided based on the patient's age, clinical manifestation, previous treatments, and treatment availability. Although very rare, GIT-metastasis of the breast cancer after a long interval (for example, up to more than 20 years) can occur: ILC is the most frequently observed. We must aware that GIT-metastasis can occur long after the initial treatment of breast cancer. This justifies prolonged surveillance for patients treated for early ILCs.

Keywords: Breast cancer, gastrointestinal tract, gastric metastasis, rectal metastasis, case report.
\end{abstract}

Copyright (C) 2021 The Author(s): This is an open-access article distributed under the terms of the Creative Commons Attribution 4.0 International License (CC BY-NC 4.0) which permits unrestricted use, distribution, and reproduction in any medium for non-commercial use provided the original author and source are credited.

\section{INTRODUCTION}

Breast cancer is the most common and the most deadly malignant tumour in women worldwide [1]. With all the diagnostic and therapeutic advances recorded in recent years, it remains that $15 \%$ of breast cancers still have distant metastases at the time of diagnosis; and at least $50 \%$ of breast cancers diagnosed early and treated curatively will relapse in metastatic mode[2,3]. Although visceral metastases of breast cancer are common, metastases in the digestive tract are unusual and rare. When they do occur, they are often linked to the lobular carcinoma histological subtype [4]. In our department, out of 3328 patients followed for breast cancer from 2012 to 2019, the case we are reporting is the first known case to have metastasized from breast cancer to the GIT. We therefore report this case of a 52 year old patient followed for infiltrating lobular carcinoma of the left breast, treated by radical mastectomy and adjuvant treatments (chemotherapy, radiotherapy and hormone therapy) in 2007, and who presented 12 years after the initial treatment, with metastases of the digestive tract, first at the gastric level in 2019, and then at the rectal level in 2020 . 


\section{PATIENT PRESENTATION}

She is a 52 year old patient, postmenopausal with no other associated medical history, followed since 2007 for an infiltrating lobular carcinoma (ILC) of the left breast which she was treated in 2007 by surgery, left mastectomy and axillary lymph node dissection. The tumor had been classified as pT2N1M0 and Scarff-Bloom and Richardson (SBR) grade II. On immunohistochemistry, lobular histology has been assessed on positivity of anticytokeratin 7 and anti-GATA 3 antibodies, negativity of anti-cytokeratin 20 antibodies and the loss of ECadherin. Moreover, tumor cells expressed hormone receptors (Estrogen receptors: 70\%, Progesterone receptors: $40 \%$ ), but were negative for the human epidermal growth factor receptor2 (HER2). The patient has benefited from adjuvant treatments based on sequential chemotherapy with 3 courses of FEC100 (5-Fluoro-Uracil: 500 mg/m2, Epirubicin: $100 \mathrm{mg} / \mathrm{m} 2$, cyclophosphamide: $500 \mathrm{mg} / \mathrm{m} 2$; Day1 = Day21) and 3 courses of Docetaxel $(100 \mathrm{mg} / \mathrm{m} 2$, Day1 = Day21), radiotherapy on the left thoracic wall and the homolateral axillary lymph node area, and endocrine therapy with Tamoxifen $20 \mathrm{mg}$ per day for a total duration of 5 years, until June 2013. Then, she was followed up regularly with radioclinical examination every six months for five years until 2018, without clinical or radiological arguments in favor of relapse. After five years of such active surveillance, a thoracic and abdominopelvic computed tomography (TAP-CT) scan was performed on April 1, 2019. It revealed a regular, non-stenosing, circumferential gastric parietal thickening measuring $13 \mathrm{~mm}$ thick, involving the entire gastric wall and extended to the antropyloric region (figure 1 ).

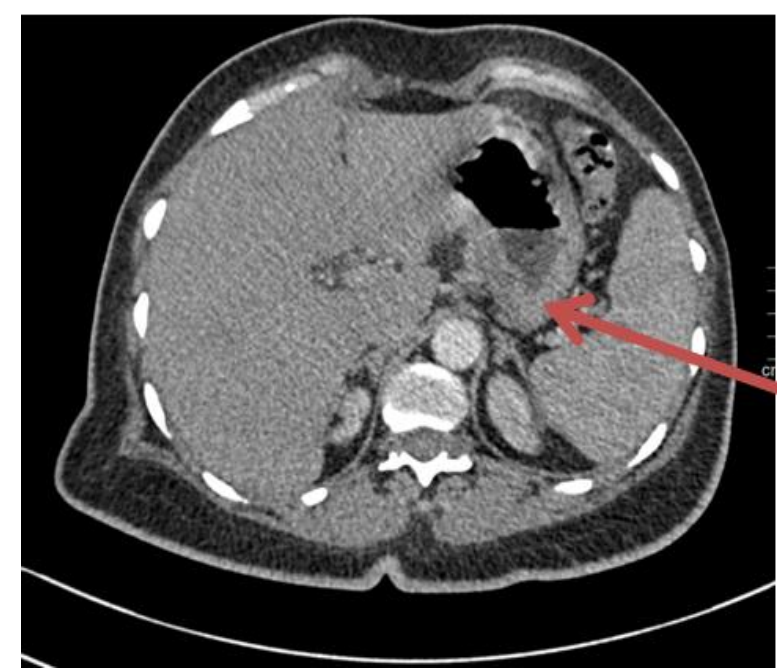

Fig-1: CT scan in axial section showing parietal thickening of gastric metastasis from breast carcinoma confirm Bone metastases were also presents as confirmed on bone scintigraphy assessment. The oesogastroduodenoscopy carried out revealed an aspect of erythematous and erosive pangastritis at the fundal level with hypertrophied folds whose histological examination suggested a gastric localization of a poorly differentiated and infiltrating carcinoma. The immunohistochemistry of the tumor cells was positive for anticytokeratin antibodies, and staining for estrogen and progesterone receptors and HER2 status showed similar results to those of the primary breast cancer and consistent with gastric metastasis of mammary origin. A first-line endocrine therapy treatment with an aromatase inhibitor, Letrozole (1 tablet of $2.5 \mathrm{mg}$ daily), combined with a bone antiresorptive drug, zoledronic acid (4 mg intravenous, 1 injection per month) was conducted since September 2019, and was well tolerated. One year later, evaluation by TAP-CT scan and bone scintigraphy noted, on the one hand, a total response at gastric level, with a stable aspect of the diffuse bone involvement. On the other hand, there was a regular and circumferential non-stenosing parietal thickening of the rectum, 6 $\mathrm{cm}$ from the anal verge measuring $10 \mathrm{~mm}$ maximum thickness and extended over $11 \mathrm{~cm}$ (figure 2).

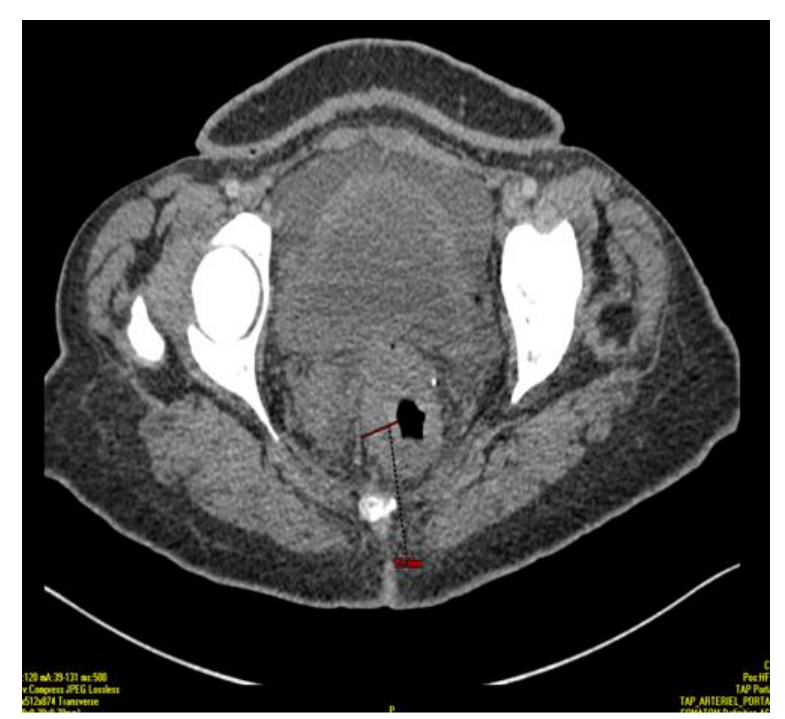

Fig-2: CT scan in parenchymal window of axial section showing parietal, circumferential and irregular thickening of the rectum metastasis from breast carcinoma

Proctosigmoidoscopy noted an erythematous and bleeding rectal mucosa. Histological analysis concluded that it was a poorly differentiated adenocarcinoma with independent cells. The immunohistochemistry of the tumor cells was positive for anti-GATA 3 antibodies, positive for anticytokeratin 7 antibodies, negative for 
Gustave Negamiyimana et al; Glob Acad J Med Sci; Vol-3, Iss- 4 (July-Aug, 2021): 129-132.

anticytokeratin 20 antibodies in favour of a rectal location of a carcinoma proliferation of mammary origin. However, there were no associated clinical symptoms, as the patient remained with a good performance status. The treatment consisted of chemotherapy with capecitabine (Xeloda) at $1000 \mathrm{mg} / \mathrm{m} 2$ twice daily for 14 days for cycles of 21 days. Radiological evaluation after 5 courses of chemotherapy revealed a partial response in the rectal lesions which had regressed by at least $40 \%$. The treatment was generally well tolerated and ongoing to date.

\section{DISCUSSION}

It is very rare for the GIT to be reported as a metastatic site for cancers, let alone breast cancer, whose most common sites of distant metastases typically involve lymph nodes, pleura, lungs, bone, liver and brain [5]. Some data from retrospective or autopsy series present cancer metastases, including breast cancer, in the GIT as a rare but possible situation [6]. In a retrospective series covering 25 years from November 1992 to August 2017, Li L. et al., were only able to collect 30 cases of gastric metastases with 5 cases, or $16 \%$, of mammary origin [7]. In an earlier series investigating 12,001 patients followed for metastatic breast cancer from 1985 to 2000 , McLemore et al found that only 41 patients, or $0.3 \%$, presented with metastases in the gastrointestinal tract [8]. The available data do not provide any particular explanation for the rarity of metastases in the GIT. The hypothesis of an adaptation problem in the GIT that does not provide metastatic tumor cells with a microenvironment conducive to their survival and proliferation could explain such rarity. Nevertheless, information concerning the real incidence of gastrointestinal metastases is probably underestimated because it is a subject most often addressed in the form of case reports or a small series of cases.

The site of breast cancer metastasis in the GIT is variable, but the stomach is the most affected site while colorectal involvement is rare $[8,9]$. Our patient presented with gastric metastasis followed by rectal metastasis 1 year later. Although more than twenty different histological subtypes are described for breast cancer, infiltrating lobular carcinoma (ILC), which is not very common (10\%), is the most likely to metastasize to the digestive tract $[4,9]$. Gastric and intestinal metastases were also due to ILC in my patient.

The time between the appearance of these gastric or intestinal metastases and the initial diagnosis of breast cancer is also variable, but metachronous metastases are the most common. However, a synchronous discovery for multimetastatic breast cancer at time of diagnosis has also been described in the literature [8]. Our patient presented with gastric metastasis 12 years after the initial diagnosis and with rectal metastasis 13 years after the initial diagnosis. In the McLemore et al series, the median time to occur was 7 years after primary breast cancer [8]. Ambroggi et al found a median duration of 9.5 years [9]. In addition, a longer interval of more than 20 years between initial diagnosis and the occurrence of metastases in the gastrointestinal tract has also been reported in the literature [10]. This justifies the need for prolonged monitoring of patients treated for early stage breast cancer, especially with ILCs histologies.

Clinical manifestation of breast cancer metastases in the gastrointestinal tract is variable, non- specific or even absent, making diagnosis difficult. The confusion with a primary digestive cancer is also quite possible. A digestive symptomatology mimicking a primitive cancer of the GIT with abdominal pain, dyspepsia, anorexia, rectal syndrome, ... has been found in other series $[8,9]$. In the case of our patient, the discovery was incidental during a CT scan to assess current treatment. Digestive endoscopy was essential for a better definition of the lesions and their location; but also, to perform biopsies for histopathological confirmation. According to the available data, gastric metastases of mammary origin produce a classic appearance of plastic linite (linitis plastica) consisting of a diffuse thickening of the walls which produces an intense fibrous reaction of the submucosa [2, 9]. At the colorectal level, presentations can have the appearance of a chronic inflammatory bowel disease such as Crohn's disease, or simply as localized nodular, polyploid or ulcerated lesions of the intestinal glandular epithelium [9]. In our case, endoscopic presentation was also non-specific, with the aspect of erythematous and erosive pangastritis with hypertrophied folds at the gastric level. At the rectal level, the metastasis presents as an erythematous and bleeding rectal mucosa on contact.

Histological diagnosis is difficult and must take into account the context of the known breast neoplasia. In all cases as for our patient, immunohistochemical analysis was essential to distinguish metastatic from primary gastrointestinal carcinoma by establishing histopathological similarities with the primary breast cancer, and thus confirm the mammary origin of the digestive malignancy. Indeed, metastatic cells of lobular 
breast cancer frequently retain the same receptor status as the primary tumor. They are generally positive for cytokeratin 7 (CK7), for GATA type 3 protein, for Gross cystic disease fluid protein 15 (GCDFP-15), and negative for cytokeratin 20 (CK20) with the inactivation of E-cadherin which is responsible for intercellular adhesion [6].

Once the diagnosis has been established, the treatment of gastrointestinal metastases should be discussed on a case-by-case basis, taking into account age, hormone receptor status, clinical presentation at the time of diagnosis and degree of urgency (such as obstruction, hemorrhage, etc.), previous treatments and treatments availability. For our patient, gastric and rectal metastases were considered as a progression of the known mammary carcinoma. Having no clinical manifestation at the time of diagnosis, treatment consisted of systemic treatment with capecitabine(Xeloda)-based chemotherapy, $1000 \mathrm{mg} / \mathrm{m} 2 \times 2$ per day for 14 days, D1 = D21. Patient's tolerance was good under treatment. Evaluation after 5 courses of chemotherapy revealed a partial response in the rectal lesions which had regressed by at least $40 \%$ (5,8mm maximum thickness vs $10 \mathrm{~mm}$ initially). To date, the treatment was ongoing.

\section{CONCLUSION}

Gastric or intestinal metastasis of breast cancer is a rare entity. However, in a patient with a history of mammary carcinoma, especially lobular carcinoma, and presenting with either new gastrointestinal disorders or newly diagnosed abnormal tumor or a radiological thickening of gastrointestinal tract, a digestive metastasis of the former breast cancer must be suspected. A thorough clinicopathological and immunohistochemical analysis allow us to differentiate between a primary digestive tumor and a metastasis of mammary origin; and to avoid under- or over-treatment in such patients. The treatment depends on the presentation at diagnosis, which may consist of surgery or systemic treatments with chemotherapy or endocrine therapy.

\section{Conflict of interest statement}

The authors declare no competing interest

\section{Patient's consent}

We certify that we have obtained the patient consent. By a consent form, the patient has given her consent for her clinical information to be reported in the journal. The patients understand that her anonymity will be guaranteed.

\section{REFERENCES}

1. Bray, F., Ferlay, J., Soerjomataram, I., Siegel, R.L., Torre, LA., et Jemal, A. (2018). Global cancer statistics 2018: GLOBOCAN estimates of incidence and mortality worldwide for 36 cancers in 185 countries. CA Cancer J Clin, 68(6):394-424.

2. Baa, A.K., Naik, R.D., Vanidassane, I., Arora, S., Shamim, A.S., Mallick, S. (2019). Unusual Gastric Metastasis in Triple-Negative (Estrogen Receptor/ Progesterone Receptor/HER2neu Negative) GATA-Binding Protein 3-Positive Breast Cancer. Indian J Nucl Med, 35(1):82-3.

3. Roché, H. et Vahdat, L.T.(2011). Treatment of metastatic breast cancer: Second line and beyond. Ann Oncol, 22(5);1000-10.

4. Korhonen, T., Kuukasjärvi, T., Huhtala, H., Alarmo, E., Holli, K., Kallioniemi, A., .(2013). The impact of lobular and ductal breast cancer histology on the metastatic behavior and long term survival of breast cancer patients. The Breast, 22(6);1119-24.

5. Soni, A., Ren, Z., Hameed, O., Chanda, D, Morgan, J.C., Siegal, G.P.(2015). Breast Cancer Subtypes Predispose the Site of Distant Metastases. Am Soc Clin Pathol, 4(143):4718.

6. Geada, L., Kantor, M., Mohan, K., Weingrad, D. et Nasiff, L.S. (2020). An Uncommon Presentation of a Common Disease: A Review of Gastric Metastasis From Breast Carcinoma. Cureus, 12(12):1-5.

7. Lin, L., Wang, X., Tang, C., et Liang, J (2019). Clinical Characteristics and Prognosis of Gastrointestinal Metastases in Solid Tumor Patients : A Retrospective Study and Review of Literatures. Anal Cell Pathol, 1-7.

8. Mclemore, E.C., Pockaj, B.A., Reynolds, C., Gray, R.J., Hernandez, J.L., Grant, C.S. (2005). Breast Cancer: Presentation and Intervention in Women With Gastrointestinal Metastasis and Carcinomatosis. Ann Surg Oncol, 12(11):886-94.

9. Ambroggi, M., Stroppa, E.M., Mordenti, P., Biasini, C., Zangrandi, A., Michieletti, E . (2012). Metastatic Breast Cancer to the Gastrointestinal Tract: Report of Five Cases and Review of the Literature. Int J ofBreast Cancer, 1-8.

10. Libânio, D., Mário, D.R., Pedro, P.N.G. (2018). Gastric Metastasis of Breast Cancer after 20 Years. Port J Gastroenterol, 25; 99-101. 